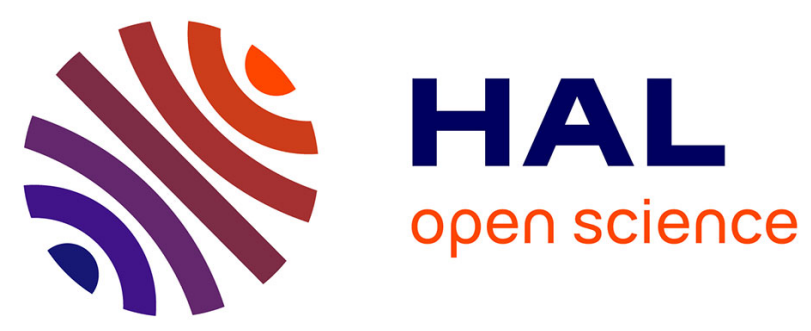

\title{
Response of a stratified boundary layer on a tilted wall to surface undulations
}

\author{
Pierre-Yves Passaggia, Patrice Meunier, Stéphane Le Dizès
}

\section{To cite this version:}

Pierre-Yves Passaggia, Patrice Meunier, Stéphane Le Dizès. Response of a stratified boundary layer on a tilted wall to surface undulations. Journal of Fluid Mechanics, 2014, 751, pp.663-684. 10.1017/jfm.2014.296 . hal-01174040

\author{
HAL Id: hal-01174040 \\ https://hal.science/hal-01174040
}

Submitted on 22 Oct 2015

HAL is a multi-disciplinary open access archive for the deposit and dissemination of scientific research documents, whether they are published or not. The documents may come from teaching and research institutions in France or abroad, or from public or private research centers.
L'archive ouverte pluridisciplinaire HAL, est destinée au dépôt et à la diffusion de documents scientifiques de niveau recherche, publiés ou non, émanant des établissements d'enseignement et de recherche français ou étrangers, des laboratoires publics ou privés. 


\title{
Response of a stratified boundary layer on a tilted wall to surface undulations
}

\author{
PIERRE-YVES PASSAGGIA ${ }^{1,2}$, PATRICE MEUNIER ${ }^{1} \dagger$ \\ and STÉPHANE LE DIZE S $\mathbf{S}^{1}$ \\ ${ }^{1}$ Aix Marseille Universit, CNRS, Centrale Marseille, IRPHE UMR 7342, 13384, Marseille, \\ France \\ ${ }^{2}$ ONERA, the French Aerospace lab F-92322 Châtillon cedex, France
}

(Received 3 April 2014)

The structure of a stratified boundary layer over a tilted bottom with a small streamwise undulation is studied theoretically and numerically. We show that the tilt of the boundary can induce strong density variations and wall-transverse velocities in the critical layer where the frequency of the forcing by the topography $k U\left(z_{c}\right)$ is equal to the transverse Brunt-Väisälä frequency $N \sin \alpha$ ( $N$ being the vertical Brunt-Väisälä frequency). The viscous solution in the critical layer, obtained and compared with direct numerical simulation results, are in good agreement for both the scaling and the spatial structure. The amplitude of the transverse velocity response is also shown to exhibit quasi-resonance peaks when the stratification strength is varied.

Key words: Stratified flow, boundary-layer flows, rough surface, critical layers.

\section{Introduction}

Stratified boundary layer flows occur in both the ocean and the atmosphere when a tidal current or a nocturnal wind develops along a surface. The topology of these surfaces is generally complex and in the case of slopes, tilted with respect to the stratification. A natural approach to account for small amplitude topology is to consider a corrugated surface. For instance, these surfaces are representative of ocean bottoms such as seamounts where strong flow acceleration along the slopes has been reported in (Genin et al. 1986). Sand waves formation, merely found along shallow continental slopes, are also a representative configuration (Besio et al. 2004). In the present work, we show that a free stream in a stratified medium on a tilted corrugated surface generates a strong transverse flow by a subtle interplay of stratification and shear.

Stratified boundary layer flows have mainly been studied on flat horizontal surfaces. Numerous works exist in the atmospheric context (see Garratt 1992). More recently, Mahrt (2014) reviewed the characteristics of the stably stratified atmospheric boundary layer. Corrugated surfaces have often been used to facilitate the boundary layer transition. There is an important piece of literature on the receptivity of boundary layers for its possible applications to aeronautics (see for instance Saric et al. 2002). In the atmospheric context, most works were motivated by understanding the effect of topography

$\dagger$ Email address for correspondence: meunier@irphe.univ-mrs.fr 
(e.g. Jackson \& Hunt 1975; Taylor et al. 1987; Gong et al. 1996; Athanassiadou \& Castro 2001), and vegetation (e.g. Finnigan et al. 2009) on the boundary layer turbulence characteristics. The influence of a stable stratification has also been considered in the experimental works of Ohya (2001) and Ohya \& Uchida (2008). On the theoretical side, Thorpe (1992) has derived the structure of the lee internal waves generated by an undulated topography over a sloping bottom, in the presence of stratification and rotation. This structure has been observed numerically by MacCready \& Pawlak (2001) over a sinusoidal topography but also over a solitary hill. Finally, one can mention the recent works of Wu \& Zhang $(2008 a, b)$ who analyzed the development of a viscous instability wave and its interaction with a bump in a stratified boundary layer using the triple-deck framework (Sykes 1978). The effect of a slope has also been considered in a few field studies (Park \& Park 2006; Nadeau et al. 2013) but we are not aware of any fundamental study in a controlled experimental framework.

When the surface is flat but inclined, we know that the deformation of the isopycnal near the boundary is the source of a flow along the slope which has been studied by Garrett et al. (1993). Candelier et al. (2012) have recently shown that an inviscid instability, different from the inflectional instability, may also be active. This instability involves a phenomenon of over-reflection (Acheson 1976; Lindzen \& Barker 1985) between the boundary and a critical point where the phase velocity matches the meanflow velocity. It is very similar to the instability observed around a rotating cylinder (Riedinger et al. 2011). In the present work, this instability, as well as the viscous boundary layer instability are not considered. We therefore assume that either the flow is stable (this will be the situation for our numerical simulations) or the forced response described below is not destroyed by these instabilities. We consider a small sinuous undulation with a fixed wavenumber. We are going to show that the wave generated by this undulation may exhibit a singular structure due to the presence of a critical point singularity.

This very thin structure is located within the boundary layer, with a large amplitude, even for small amplitudes undulations, which is very likely to be an important source of mixing and transport for ocean and atmospheric flows over slopes.

The paper is organized as follows. In $\S 2$, we introduce the framework with the flow configuration and the numerical procedure. In $\S 3$, we first provide numerical results. These results are then interpreted using an asymptotical analysis for large Reynolds numbers and small undulation amplitudes which leads to an expression for the maximum transverse velocity. The dependence of this expression with respect to the Froude number and the undulation wavenumber is further analyzed using a small Froude number description. Details of the analysis are given in an appendix. Section $\S 4$ provides a brief summary

of the results and tentative applications to oceanic currents and atmospheric boundary layers.

\section{Problem formulation}

\subsection{Flow configuration}

We consider a boundary layer flow in a stratified fluid on a sinusoidally deformed tilted wall, as shown in Fig. 1. The wall is globally tilted in the cross-stream direction such that the $z^{*}$-axis of the $\left(x^{*}, y^{*}, z^{*}\right)$ frame attached to the wall makes an angle $\alpha$ with respect to the $Z^{*}$-direction of stratification. The tilted frame of reference is obtained from the horizontal/vertical coordinates $(X *, Y *, Z *)$ using the change of variables:

$$
x^{*}=X, \quad y^{*}=Y \cos (\alpha)+Z \sin (\alpha) \text { and } z^{*}=Z \cos (\alpha)+Y \sin (\alpha) .
$$


The inlet flow is assumed to be a typical tanh boundary layer flow of thickness $\delta^{*}$ and free stream velocity $U_{\infty}^{*}$ :

$$
\mathbf{U}^{*}=U_{\infty}^{*} \tanh \left(\frac{z^{*}+z_{0}^{*}}{\delta^{*}}\right) \mathbf{e}_{\mathbf{x}} .
$$

A small penetration length $z_{0}^{*}$ has been introduced to authorize the flow to slide on the boundary with a velocity $U_{0}^{*}=U_{\infty}^{*} \tanh \left(z_{0}^{*} / \delta^{*}\right)$. This boundary condition mimics the condition obtained on the top of a viscous sublayer where roughness or small scale inhomogeneities could be present. It is the simplest way to model the flow over a canopy.

The fluid is assumed stably stratified along the vertical $Z^{*}$ direction with a constant buoyancy frequency

$$
N^{*}=\sqrt{-\left(g / \rho_{0}^{*}\right)\left(\partial \bar{\rho}^{*} / \partial Z^{*}\right)}
$$

where $g$ is gravity.

The boundary is defined by the $2 \mathrm{D}$ surface:

$$
z^{*}=\eta^{*}\left(x^{*}\right)=h^{*} \sin \left(k^{*} x^{*}\right)
$$

where $h^{*}$ and $k^{*}$ correspond to the amplitude and wavenumber of the topography, respectively.

The flow is governed by the Navier-Stokes equations under the Boussinesq approximation, the incompressibility condition and the advection-diffusion equation for the density

$$
\begin{aligned}
\frac{D \mathbf{u}^{*}}{D t} & =-\frac{\nabla p^{*}}{\rho_{0}^{*}}-\frac{\rho^{*}}{\rho_{0}^{*}} g \mathbf{e}_{\mathbf{z}}+\nu \Delta \mathbf{u}^{*} \\
\nabla \cdot \mathbf{u}^{*} & =0 \\
\frac{D \rho^{*}}{D t} & =\kappa \Delta \rho^{*},
\end{aligned}
$$

where $\nu$ and $\kappa$ are the kinematic viscosity and the thermal diffusivity respectively. It satisfies the following boundary conditions at the wall

$$
\left.\mathbf{u}^{*}\right|_{z^{*}=\eta^{*}}=U_{0}^{*} \mathbf{t} ;\left.\quad \nabla \rho \cdot \mathbf{n}\right|_{z^{*}=\eta^{*}}=0,
$$

where $\mathbf{t}$ and $\mathbf{n}$ are the wall tangent and normal vectors respectively. Far from the wall $\left(z^{*} \rightarrow \infty\right)$, the velocity and density fields are assumed to satisfy

$$
\mathbf{u}^{*} \sim U_{\infty}^{*} \mathbf{e}_{\mathbf{x}} ; \quad \rho^{*} \sim \bar{\rho}^{*}=\rho_{0}^{*}\left(1-\left(z^{*} \cos \alpha+y^{*} \sin \alpha\right) N^{* 2} / g\right) .
$$

In the following, we non-dimensionalize all the variables using $\delta^{*}, U_{\infty}^{*}$ and $\rho_{0}^{*}$, and denote these variables without the star. The problem is characterized by 6 non-dimensional parameters

$$
R e=\frac{U_{\infty}^{*} \delta^{*}}{\nu}, \quad F=\frac{U_{\infty}^{*}}{N^{*} \delta^{*}}, \quad \alpha, \quad h=h^{*} / \delta^{*}, \quad k=k^{*} \delta^{*}, \quad \operatorname{Pr}=\frac{\nu}{\kappa}, \quad U_{0}=\frac{U_{0}^{*}}{U_{\infty}^{*}} .
$$

We are interested in the configurations where $R e$ is large and $h$ is small. We also implicitly assume that the boundary layer flow on the undeformed wall is stable. This means that the Reynolds number is below the critical Reynolds number for the appearance of unstable Tollmien-Schlichting modes and unstable radiative modes.

\subsection{Numerical procedure}

Since the inlet flow is two-dimensional, we can assume that the flow remains twodimensional downstream that is independent of the cross-stream variable $y$. For most 


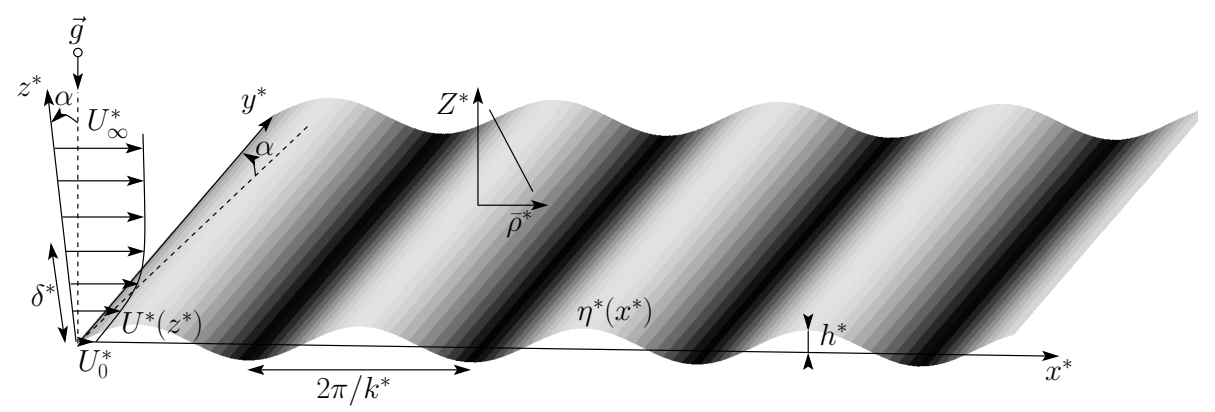

FiguRE 1. Sketch of the flow geometry

numerical simulations, the numerical computational domain is taken from $x=-7.5$ to 52.5 and from $z=\eta(x)$ to $H=18$. However, the boundary deformations only extend from $x=0$ to $x=42.5$, as shown in Fig. 2(b). The infinite flow domain is transformed into a Cartesian one using the mapping $\bar{x}=x, \quad \bar{z}=z-\eta(x)$, the barred coordinates being the computational ones. The numerical procedure is detailed in Marquillie \& Ehrenstein (2002) and proved to accurately predict transitional boundary-layers phenomenon (Marquillie \& Ehrenstein 2003; Passaggia et al. 2012). Since the mapping transformation requires $\eta(x)$ to be twice differentiable, the lower boundary has been smoothed in the vicinity of $x=0$ and $x=42.5$ using regularization procedures based on convolution between the wall function $\eta(x)$ and a third order polynomial kernel. The inlet boundary condition is given by the boundary layer profile (2.2) and $\rho=\bar{\rho}$.

Following the numerical procedure of Marquillie \& Ehrenstein (2003), a convective condition has been implemented at the outflow for all velocity components $\mathbf{u}$ and the density $\rho$ such that

$$
\frac{\partial \mathbf{u}}{\partial t}=U_{c} \frac{\partial \mathbf{u}}{\partial x}, \quad \frac{\partial \rho}{\partial t}=U_{c} \frac{\partial \rho}{\partial x}, \quad U_{c}=\frac{1}{z^{\dagger}} \int_{0}^{z^{\dagger}} u_{x}(42.5, z) \mathrm{d} z
$$

where $z^{\dagger}$ was chosen for the streamwise velocity $u_{x}(42.5, z)$ to be equal to 0.5 , which is the uniform flow at infinity (Marquillie \& Ehrenstein 2002).

When addressing the question of stratified incompressible flows in a finite computational domain, sponge layers appear to be mandatory to avoid spurious modes arising from a finite computational domain together with boundary conditions (2.9). In the present case, the sponge layers consist of hyperbolic tangent functions which smoothly cancel the advection term of the equation for the density $\rho$. In the present study, they are present close to the inlet for $-7.5<x<-4.5$ and far from the wall for $15<z<18$, which has proved to be appropriate to achieve convergence in all cases considered. The present calculations have been performed calculating first the vertical flow solution at $\alpha=90^{\circ}$. Restarting from this solution, the tilted flow solution has been computed until convergence of the time marching algorithm up to $10^{-6}$ of the solution vector.

Most numerical simulations are carried out in the computational domain defined above. The Reynolds number $R e$ is varied from 60 to 1200, the Froude number $F$ from 0.95 to 1.15, the tilt angle $\alpha$ from 15 to 75 degrees and the non-dimensional wavenumber $k$ from 0.85 to 1.25. Different computational domains have also been considered in order to treat more extreme values of $k$ and document the behabiour of the solution for small and large Froude numbers. In all the simulations, the sliding velocity is fixed to $U_{0}=0.1$ and the Prandtl number $\operatorname{Pr}$ is equal to 0.7 in order to simulate the thermal diffusion of air. 


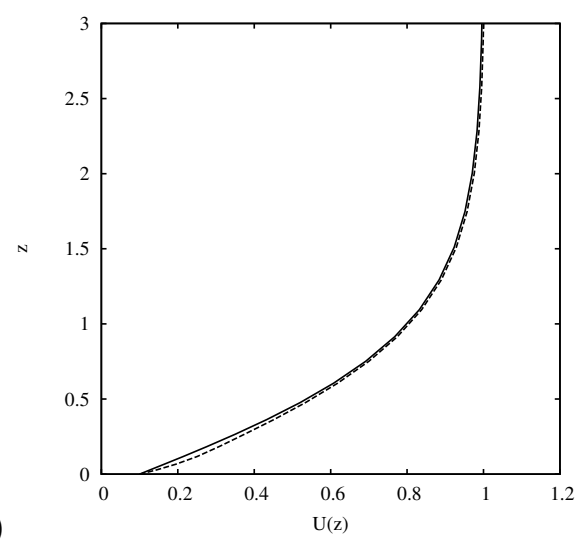

(a)

FiguRE 2. Inlet (solid line) and at the summit of an oscillation $x=30.3$ (dashed line) velocity profiles (a) at $R e=593$ and $h=0.06$. Graph of the lower boundary $\eta(x)$ (b) of the small computational domain at $h=0.06$.

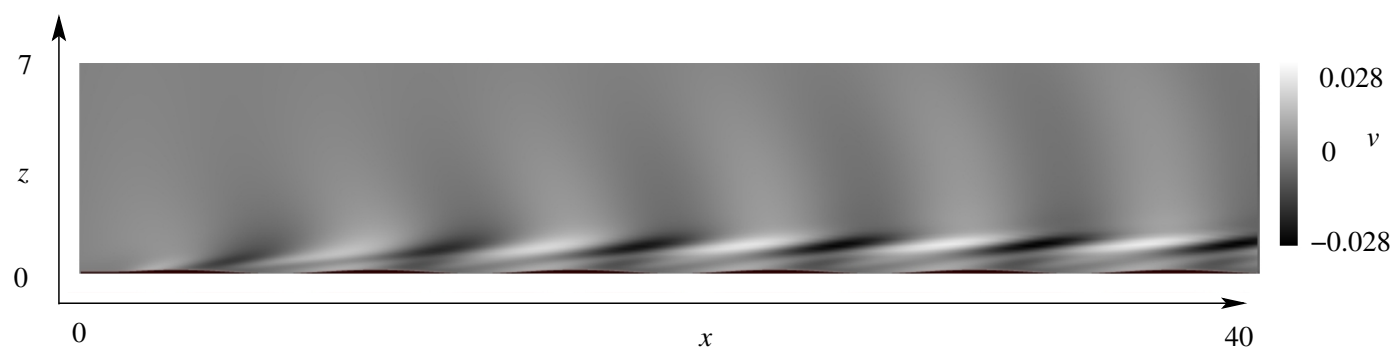

FiguRE 3. 2D field of the transverse velocity $v(x, z)$ for $h=0.06, F=1.046, k=1.041$, $R e=1186$ and $\alpha=\pi / 4$.

\section{Structure of a stratified tilted boundary layer}

\subsection{Numerical results}

In this section, we present the typical characteristics of the flow obtained in the numerical simulations. Figure 3 shows the transverse component of velocity in a plane normal to the bottom. The field contains a series of alternate lobes clearly separated from the bottom with the same wavelength as the undulated topography, but in phase quadrature. Above these lobes, the velocity presents almost vertical bands of weak amplitude, characteristic of lee waves created by an undulated topography.

Figure 4 shows the profile of velocity and density at the altitude of the lobes $z_{c}=0.75$. It is striking to see that the transverse velocity (dashed line) is 5 times larger than the normal velocity although the sliding velocity along the topography generates only a normal velocity. The density also exhibits large sinusoidal perturbations which are 10 times larger than the normal velocity. These oscillations start at the beginning of the undulated topography $(x=0)$, grow during a transient stage of about 3 to 4 undulations and then saturate.

The altitude $z_{c}$ of the divergence of $v$ and $\rho$ has been measured for various parameters. As shown in Fig. 5, it is independent of the height of the topography and very weakly dependent on the Reynolds number. However, it clearly depends on the Froude number, the tilt angle and the wavenumber. This critical altitude $z_{c}$ corresponds to the position where the normal component of the wavevector diverges. Indeed, in 


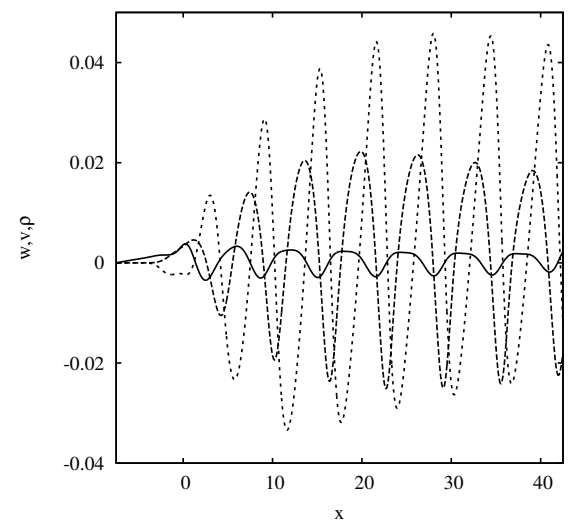

FigURE 4. Streamwise profile of the normal velocity $w$ (solid line), transverse velocity $v$ (dashed line) and density $\rho$ (dotted line) at $z_{c}=0.75 . R e=593, F=1.046, k=1.041, \alpha=\pi / 4$ and $h=0.06$

the tilted frame of reference $(x, y, z)$, a lee wave with wavevector $\mathbf{k}=(k, 0, m)$ has a frequency given by the linear dispersion relation:

$$
\omega=k U \pm \frac{1}{F} \frac{\left\|\mathbf{k}_{\text {Hor. }}\right\|}{\|\mathbf{k}\|}=k U \pm \frac{\sqrt{k^{2}+m^{2} \sin ^{2} \alpha}}{F \sqrt{m^{2}+k^{2}}}
$$

The variation of $U$ with the altitude bends the waves such that the normal wavenumber $m$ varies with the altitude in order for the wave to be stationary. Assuming $\omega=0$ in (3.1) leads to

$$
m^{2}=k^{2} \frac{1-k^{2} U^{2} F^{2}}{k^{2} U^{2} F^{2}-\sin ^{2} \alpha}
$$

which diverges for

$$
U\left(z_{c}\right)=\frac{\sin \alpha}{F k}
$$

This criterion defines a critical altitude $z_{c}$ which is plotted in Fig. 5 as a solid line. It is reasonably close to the altitude where $v$ and $\rho$ diverge. This indicates that the strong variations of $v$ and $\rho$ come from the divergence of the wavenumber $m$. However, this analysis is only valid when the wavelength of the lee wave is much smaller than the thickness of the boundary layer. In the next section, the analysis is extended for any wavelength, but for the case of small undulation height $h$.

\subsection{Expansion for small wall oscillations amplitudes}

The structure of the boundary layer can be described asymptotically for a small amplitude $h$ of the topography and a large Reynolds number. The solution is decomposed into a base flow $\left(\mathbf{U}_{b}, \rho_{b}, p_{b}\right)$ and a small perturbation $(\mathbf{u}, \rho, p)$ which is searched as a spatial Fourier mode of wavenumber $k$ and amplitude $h$ :

$$
(\mathbf{u}, \rho, p)=\frac{1}{2} h k\left(\tilde{\mathbf{u}}, U_{\infty}^{2} /(\delta g) \tilde{\rho}, \tilde{p}\right) e^{i k x}+c . c .
$$

The base flow corresponds to the solution on a flat but inclined wall. In the limit of large Reynolds numbers, it remains close to the inlet flow as long as we consider streamwise location small compared to the viscous diffusion scale $x_{v}=O(R e)$. The normal flow 


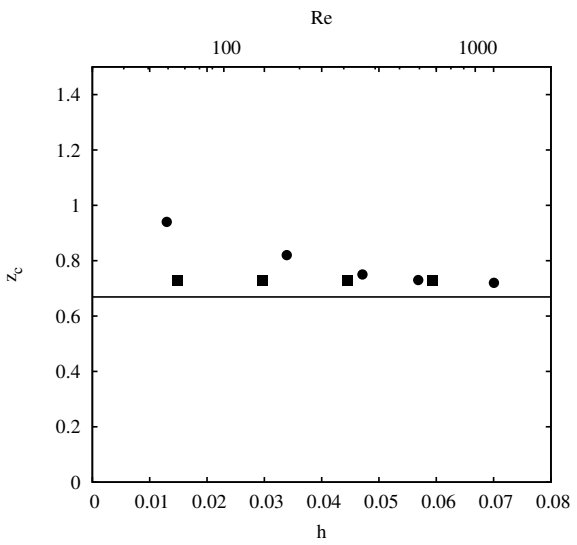

(a)

Figure 5. Position of the critical layer $z_{c}$ measured at the maximum of $v$. In (a), $z_{c}$ is plotted as a function of $h(\boldsymbol{\square})$ and as a function of $\operatorname{Re}(\boldsymbol{\bullet})$ for $F=1.046, \alpha=\pi / 4$ and $k=1.041$. In (b), $z_{c}$ is plotted as a function of $\sin \alpha / k F$ for $R e=593$ and $h=0.06$. In (b), symbols correspond to variations of $F(\boldsymbol{\square}), k(\bullet)$ and $\alpha(\mathbf{\Delta})$ and the solid line corresponds to the velocity profile $U\left(z_{c}\right)$.

correction induced by this weak diffusion process is $\mathrm{O}(1 / R e)$. A weak cross flow is also present as soon as the wall is inclined. This flow is associated with density correction generated by the inclination of the isopycnals with respect to the boundary. To cancel the normal density gradient at the wall, a density correction and a weak cross flow is created very close to the wall. This boundary layer flow has been analyzed in details in Garrett et al. (1993). Neither the cross flow, nor the density correction have an impact far from the boundary.

The equations satisfied by the amplitude of the Fourier mode can be obtained by linearizing the governing equations (2.5) around the base flow solution. We get at first order in $h$ :

$$
\begin{aligned}
i k U \tilde{u}+\tilde{w} U^{\prime} & =-i k \tilde{p}+\frac{1}{R e} \tilde{\Delta} \tilde{u} \\
i k U \tilde{v} & =-\sin \alpha \tilde{\rho}+\frac{1}{R e} \tilde{\Delta} \tilde{v} \\
i k U \tilde{w} & =-\cos \alpha \tilde{\rho}-\frac{\partial \tilde{p}}{\partial z}+\frac{1}{R e} \tilde{\Delta} \tilde{w} \\
i k U \tilde{\rho} & =\frac{\tilde{w} \cos \alpha+\tilde{v} \sin \alpha}{F^{2}}+\frac{1}{\operatorname{RePr}} \tilde{\Delta} \tilde{\rho} \\
i k \tilde{u}+\frac{\partial \tilde{w}}{\partial z} & =0,
\end{aligned}
$$

where $\tilde{\Delta}=\partial_{z}^{2}-k^{2}$. Note that we have artificially kept small diffusion terms but neglected the base flow corrections mentioned above. We will see below that the diffusion terms do become important close to the critical layer singularity while the base flow corrections remain negligible. The boundary conditions obtained from (2.6) are at leading order in $h$

$$
\tilde{u}(z=0)=i\left(1-U_{0}^{2}\right) ; \quad \tilde{v}(z=0)=0 ; \quad \tilde{w}(z=0)=U_{0} ; \quad \partial_{z} \tilde{\rho}(z=0)=0 .
$$

Far from the wall, as $z$ goes to infinity, the perturbation should either vanish or be an outgoing wave.

In the inviscid regions where the diffusion terms are negligible, we get from (3.5) as 
$R e \rightarrow \infty$

$$
\tilde{p}=\frac{U}{i k} \frac{\partial \tilde{w}}{\partial z}-\tilde{w} \frac{U^{\prime}}{i k}, \quad \tilde{\rho}=\frac{i k U \tilde{w} \cos \alpha}{\sin ^{2} \alpha-k^{2} U^{2} F^{2}} .
$$

Introducing these expressions into (3.5c) leads to a single differential equation for the wall-normal velocity

$$
\tilde{w}^{\prime \prime}-\frac{U^{\prime \prime}}{U} \tilde{w}-k^{2} \frac{1-k^{2} U^{2} F^{2}}{\sin ^{2} \alpha-k^{2} U^{2} F^{2}} \tilde{w}=0 .
$$

This equation reduces to the well-known Taylor-Goldstein equation for the tilt angle $\alpha=0$. It is clear that the last term of this equation diverges at the point $z_{c}$ where $k U\left(z_{c}\right) F=\sin \alpha$. Such a singularity corresponds to a so-called critical point. Viscous effects have to be re-introduced to smooth the singularity. The singularity is weak as an expansion of the coefficients in powers of $z-z_{c}$ indicates that $\tilde{w}$ expands as $\tilde{w} \sim a^{ \pm}+a^{ \pm} \kappa\left(z-z_{c}\right) \log \left|z-z_{c}\right|+b^{ \pm}\left(z-z_{c}\right)$ with $\kappa=k \cos ^{2} \alpha /\left(2 \sin \alpha F U^{\prime}\left(z_{c}\right)\right)$ on either side of $z_{c}$. This critical point singularity is the classical singularity found in the stability of homogeneous shear flows (Lin 1955). Note that it is different from that of stratified shear flows over horizontal walls. The viscous smoothing can be used to get the adequate jumps conditions across the critical point:

$$
a^{+}=a^{-}=\tilde{w}_{c} ; \quad b^{+}=b^{-}+i \pi \kappa \tilde{w}_{c} .
$$

These jump conditions can also be obtained from the property that the inviscid solution remains asymptotically valid in any contour that avoids the critical point in the lower complex half plane, a condition which is derived from the asymptotic behavior of the viscous solutions near $z_{c}$ or from the condition of causality (see Lin 1955, for more explanations on this issue). These conditions tell us that the solution is continuous at $z_{c}$ but its derivative exhibits a jump.

Using these jumps conditions, equation (3.8) with the boundary conditions prescribed above can easily be solved using classical integration softwares. We get the solution which has been plotted in Fig. 6(a). Note that the jump of $\tilde{w}^{\prime}$ at $z_{c}$ is clearly visible on the real part of the signal. In this figure, the real part and the imaginary part of the solution are compared to the numerical signals of the normal velocity at the lee side $(k x=7 \pi)$ and at the bottom $(k x=7.5 \pi)$ of the undulation, respectively. We can note that except in the wall boundary layer there is a good qualitative agreement between the two results.

The inviscid approximation of the normal velocity is continuous at $z_{c}$. By contrast, equations (3.7) and (3.5b) demonstrate that both the transverse velocity $v$ and $\rho$ diverge at $z=z_{c}$. This behavior is in agreement with the very large amplitudes of the transverse velocity and of the density observed in the numerics. In order to determine the maximum amplitude of these two quantities, we have to solve the viscous critical layer. This is done in the next section.

\subsection{Viscous critical layer analysis}

The analysis of viscous critical layers is a classical problem which is described in several textbooks (see for instance Drazin \& Reid 1981). A very similar analysis has also been done in the context of vortices in Boulanger et al. (2007). We provide here the main steps of the analysis.

In the viscous critical layer, the structure of the solution is obtained by introducing a new local viscous scale $\hat{z}=\left(z-z_{c}\right) R e^{1 / 3}$. The form of the solution can be obtained by expanding the non-viscous solution close to the critical point. We obtain 


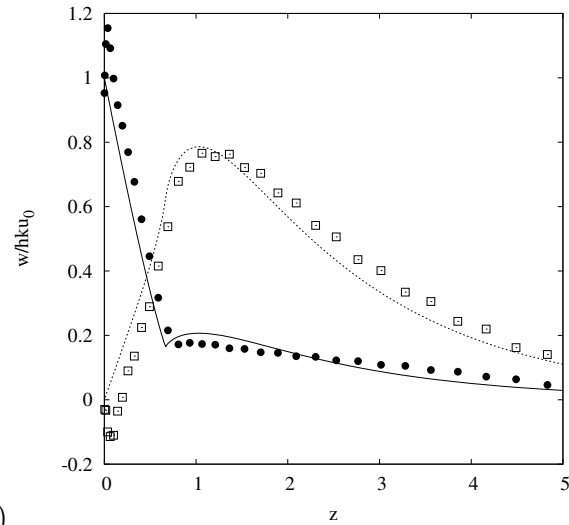

(a)

FiguRE 6. Normal profile of (a) normal velocity $w$ and (b) transverse velocity $v$. The profiles are measured on the lee side of the undulations at $x=7 \pi / k(\bullet$, solid line) and on the bottom of the undulation at $x=7.5 \pi / k$ ( $\square$, dashed line). Symbols correspond to numerical simulations and lines to theoretical predictions. $h=0.03, F=1.046, k=1.041, R e=1423$ and $\alpha=\pi / 4$.

that the solution has to be searched as

$$
\begin{aligned}
\tilde{u} & =\hat{u}(\hat{z})+\log (\boldsymbol{R} \boldsymbol{e}) \hat{u}_{s}, \\
\tilde{v} & =\hat{v}(\hat{z}) \boldsymbol{R} \boldsymbol{e}^{1 / 3}, \\
\tilde{w} & =\tilde{w}_{c}+\hat{w}(\hat{z}) \boldsymbol{R} \boldsymbol{e}^{-1 / 3}+\boldsymbol{R} \boldsymbol{e}^{-1 / 3} \log (\boldsymbol{R} \boldsymbol{e}) \hat{w}_{s} \hat{z}, \\
\tilde{\rho} & =\hat{\rho}(\hat{z}) \boldsymbol{R} \boldsymbol{e}^{1 / 3}, \\
\tilde{p} & =\hat{p}(\hat{z})+\log (\boldsymbol{R} \boldsymbol{e}) \hat{p}_{s} .
\end{aligned}
$$

Note that the $\log (R e)$ terms come from the special behavior of the normal velocity close to the critical point. Expanding the base flow velocity as

$$
U(z) \sim U\left(z_{c}\right)+U^{\prime}\left(z_{c}\right)\left(z-z_{c}\right)=\frac{\sin \alpha}{k F}+U_{c}^{\prime} \hat{z} \boldsymbol{R} e^{-1 / 3}
$$

the system $(3.5)$ reduces to

$$
\begin{aligned}
i k U_{c}\left(\hat{u}+\log (\boldsymbol{R e}) \hat{u}_{s}\right)+\hat{w}_{c} U_{c}^{\prime} & =i k\left(\hat{p}+\log (\boldsymbol{R} \boldsymbol{e}) \hat{p}_{s}\right) \\
i k U_{c} \tilde{w}_{c} & =-\cos \alpha \hat{\rho} \boldsymbol{R} \boldsymbol{e}^{1 / 3}-\frac{\partial \hat{p}}{\partial \hat{z}} \boldsymbol{R} \boldsymbol{e}^{1 / 3} \\
\frac{i \sin \alpha \hat{v} \boldsymbol{R} \boldsymbol{e}^{1 / 3}}{F}+i k U_{c}^{\prime} \hat{z} \hat{v} & =-\sin \alpha \hat{\rho} \boldsymbol{R} \boldsymbol{e}^{1 / 3}+\hat{v}^{\prime \prime} \\
\frac{i \sin \alpha \hat{\rho} \boldsymbol{R} \boldsymbol{e}^{1 / 3}}{F}+i k U_{c}^{\prime} \hat{z} \hat{\rho} & =\frac{\left(\hat{v} \sin \alpha \boldsymbol{R} \boldsymbol{e}^{1 / 3}+\cos \alpha \tilde{w}_{c}\right)}{F^{2}}+\frac{1}{\boldsymbol{P r}} \hat{\rho}^{\prime \prime} \\
i k\left(\hat{u}+\log (\boldsymbol{R e}) \hat{u}_{s}\right) & =-\frac{\partial \hat{w}}{\partial \tilde{z}}-\log (\boldsymbol{R e}) \hat{w}_{s} .
\end{aligned}
$$

At leading order in $R e^{-1 / 3}$, Eqs. (3.11c) and (3.11d) both indicate that $\hat{\rho}=$ $-i \hat{v} / F$. Introducing this result in (3.11c)-iF(3.11d) leads to a differential equation at first order for the transverse velocity $\hat{v}$

$$
2 i k U_{c}^{\prime} \hat{z} \hat{v}=\left(1+\frac{1}{P \boldsymbol{r}}\right) \hat{v}^{\prime \prime}+\frac{i \tilde{w}_{c} \cos \alpha}{F} .
$$

The function $\hat{p}(\hat{z})$ can then be deduced from (3.11b), which then gives $\hat{u}(\hat{z})$ 
from (3.11a) and $\hat{w}(\hat{z})$ from (3.11e) provided that $\hat{w}_{s}=-i k \hat{u}_{s}=-i k \hat{p}_{s} / U_{c}($ such that the log terms disappear). The matching with the outer solution gives $\hat{w}_{s}=-\tilde{w}_{c} \kappa / 3$.

Equation (3.12) is an inhomogeneous Airy equation. In order to match an inviscid solution in the outer region, the solution must behave as $\hat{z}^{-1}$ for large $|\hat{z}|$. As shown by Drazin \& Reid (1981), the solution which satisfies this condition is a generalized Airy function $B_{k}(K \hat{z}, 1)$. It can also be expressed in terms of the Scorer's function $\operatorname{Hi}(z)$ (Abramowitz \& Stegun 1965, p. 448) which verifies $\operatorname{Hi}^{\prime \prime}(z)-z \operatorname{Hi}(z)=1 / \pi$ as follows

$$
\hat{v}(\hat{z})=\frac{i \tilde{w}_{c} \pi \cos \alpha}{\xi^{2} F(1+1 / P r)} \operatorname{Hi}(-i \xi \hat{z}) .
$$

with $\xi=\left(\frac{2 k U_{c}^{\prime}}{1+1 / P r}\right)^{1 / 3}$. This expression is plotted in Fig. 6(b) as a solid line for the real part and a dashed line for the imaginary part. We can see that these two profiles are comparable to the numerical results obtained at the lee side and at the bottom of the undulation. A small shift is nevertheless observed which might come from higher order corrections.

The maximum of the transverse velocity $v_{\max }$ over the whole field can now be predicted from the maximum of the Scorer's function $\mathrm{Hi}_{\max }=\mathrm{Hi}(0) \approx 0.41$. In terms of the initial variables, we get

$$
v_{\max }=\frac{h k U_{0} R e^{1 / 3}}{(1+1 / \operatorname{Pr})^{1 / 3}} V\left(F, k, \alpha, U_{0}\right)
$$

where

$$
V\left(F, k, \alpha, U_{0}\right)=\frac{\operatorname{Hi}_{\max } \pi \cos \alpha}{F(2 k)^{2 / 3}\left(1-\sin ^{2} \alpha /(k F)^{2}\right)^{2 / 3}} \Delta_{c},
$$

with

$$
\Delta_{c}=\left|\frac{\tilde{w}_{c}}{\tilde{w}_{0}}\right|,
$$

In the above expression, we have used the relation $U_{c}^{\prime}=1-\sin ^{2} \alpha /(k F)^{2}$ and the definition $\tilde{w}_{0}=\tilde{w}(z=0)=U_{0}$.

The function $v_{\max }$ is plotted and compared to the numerical results in Fig. 7. It clearly shows that the amplitude of the critical layer is linear in $h$ with an excellent quantitative prediction of the amplitude. The amplitude also scales as $R e^{1 / 3}$ although the lowest Reynolds number is slightly above the theoretical prediction. This is possibly due to the modification of the mean profile by viscous effects in the numerics or to the fact that the critical layer becomes so large that it interacts with the near wall boundary layer flow.

The thickness $\zeta$ of the critical layer has also been measured in the numerics as the distance between the maximum and the minimum of $v$ in the shear profile (at $k x=7.5 \pi$ ). Note that each value of the thickness $\zeta$ was rescaled with the local thickness of the boundary layer, for each value of $R e$. The data is plotted in figure 8 . We observe that the thickness does decrease as $R e^{-1 / 3}$ and is in good agreement with the theory although it is $20 \%$ below the prediction.

\subsection{Dependence with respect to the Froude number and the undulation wavenumber}

In this section, we use the theory to discuss the dependence of $v_{\max }$ with respect to the Froude number and the undulation wavenumber.

The contours of the function $V\left(F, k, \alpha, U_{0}\right)$ appearing in (3.14) are plotted in Fig. 9 for a small and a large tilt angle $(\alpha=\pi / 60$ and $\alpha=\pi / 6)$. These plots show that $V$ exhibits complex variations with respect to the parameters. Note in particular in figure $9(\mathrm{~b})$ the 


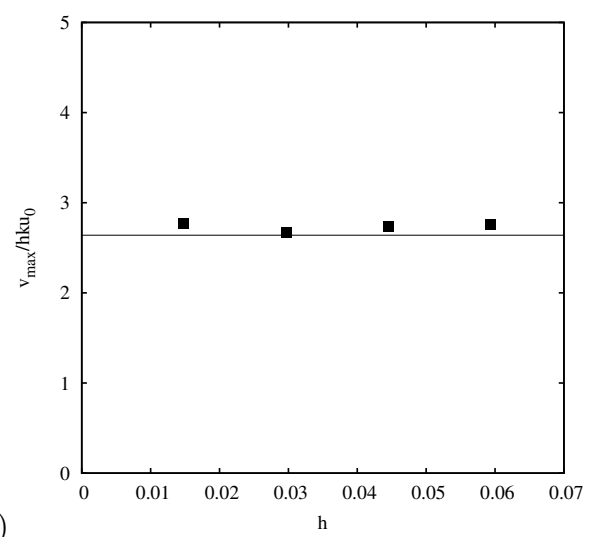

(a)

FiguRE 7. Renormalised amplitude of the critical layer $v_{\max } /\left(h k U_{0}\right)$ as a function of (a) the height of the hills $h$ and (b) the Reynolds number. Numerical values (ם) obtained at $x=7.5 \pi / k$ are compared with the theoretical prediction (solid line) given by (3.14). $F=1.046, k=1.041$ and $\alpha=\pi / 4$. In (a) $R e=593$ and in (b) $h=0.03$.

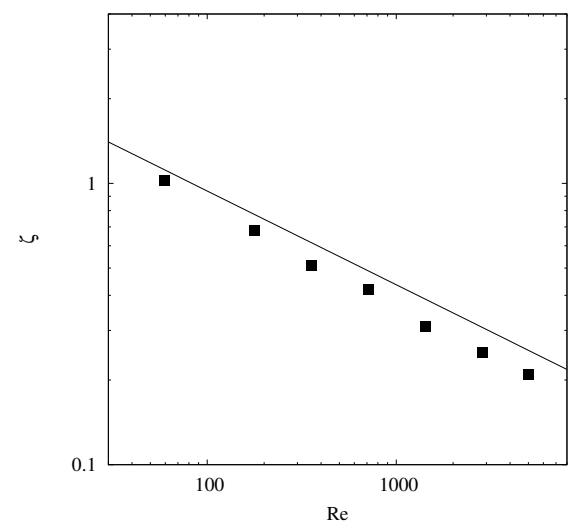

FigURE 8. Thickness of the critical layer as a function of the Reynolds number measured on the lee side of a hill at $x=7.5 \pi / k$. The theoretical prediction (solid line) is compared to the numerical simulations $(\mathbf{\square}) . h=0.03, F=1.046, k=1.041$ and $\alpha=\pi / 4$.

discontinuous behavior across the line $k F=1$ and the peaky structure for small $F$ close to the right frontier corresponding to $k F=\sin \alpha / U_{0}$. Large values of $V$ are reached in two limits which can be analyzed separately: (1) large $F$ and $k F$ close to $\sin \alpha$ (top left corners of figure 9), (2) small $F$ and $k F$ close to $\sin \alpha / U_{0}$ (bottom right corner of figure $9)$.

The first limit is the easier to analyze. For large $F$, the solution $\tilde{w}$ has indeed a simple approximation in the bulk $\dagger \tilde{w}(z) \sim U(z)$ so

$$
\Delta_{c}=\left|\frac{\tilde{w}_{c}}{\tilde{w}_{0}}\right| \sim \frac{\sin \alpha}{k F U_{0}}, \text { as } F \rightarrow \infty .
$$

$\dagger$ This approximation is valid up to the (large) altitude where the second term in (3.8) becomes of same order as the third term. 


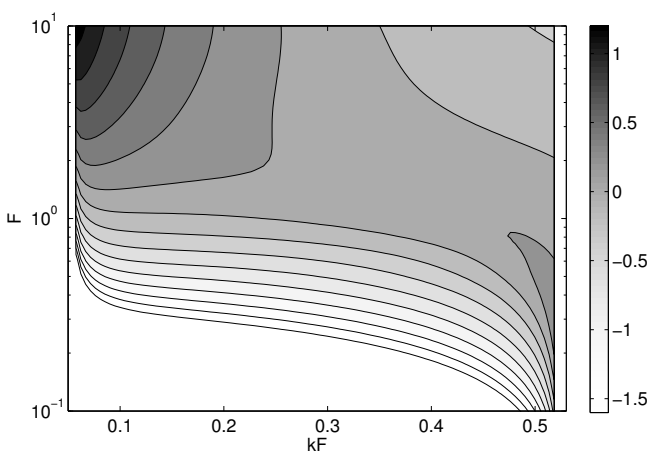

(a)

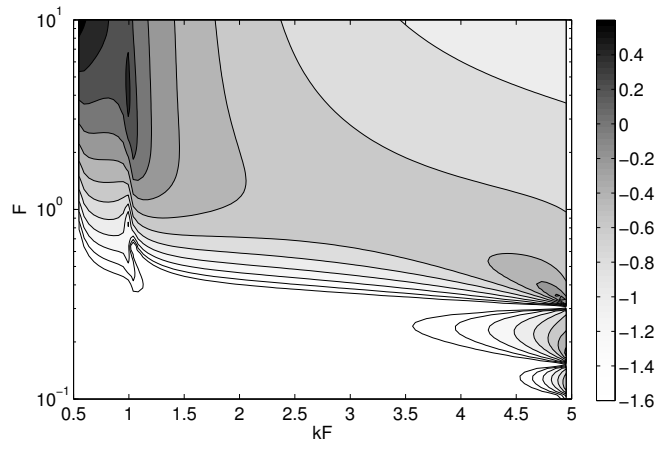

(b)

Figure 9. Contour levels of the function $\log _{10} V$ in the $(k F, F)$ plane for $U_{0}=0.1$ and (a) $\alpha=\pi / 60$ or (b) $\alpha=\pi / 6$. The function $V$ is defined for $\sin \alpha<k F<\sin \alpha / U_{0}$ only (condition of existence of a critical point).

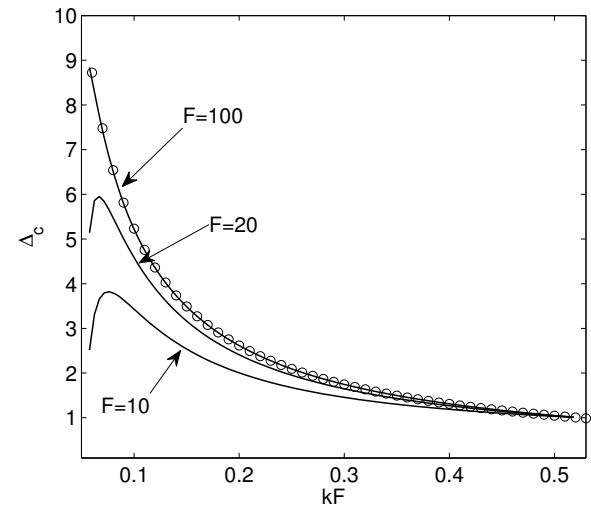

(a)

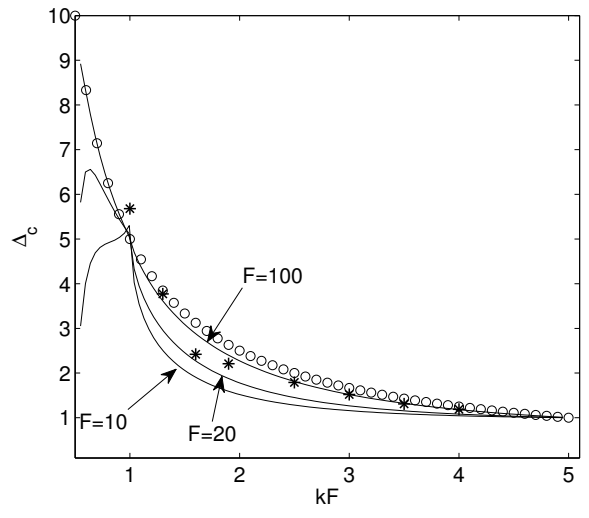

(b)

Figure 10. The function $\Delta_{c}$ versus $k F$ for different values of $F$ for (a) $\alpha=\pi / 60$ and (b) $\alpha=\pi / 6$. Solid lines: numerically computed values; Symbols: expression (3.17). The stars in (b) are estimates obtained from the direct numerical simulation for $F=10, R e=593$ and $h=0.12$.

We can then deduce that

$$
V \sim \frac{\operatorname{Hi}_{\max } \pi \cos \alpha}{U_{0} F^{1 / 3}(2 \sin \alpha)^{2 / 3}\left(1-\sin ^{2} \alpha /(k F)^{2}\right)^{2 / 3}} \quad \text { as } F \rightarrow \infty,
$$

which tell us how $v_{\max }$ varies with respect to $k F$ for large $F$. Figure 10 demonstrates that (3.17) provides a very good estimate of $\Delta_{c}$ as soon as $F$ is larger than a few tens. In figure 10(b), we have also added values of $\Delta_{c}$ obtained from the direct numerical simulation for $F=10, R e=593$ and $h=0.12$. We can see that they follow reasonably well the theoretical curve.

The second limit is more involved due to the singular structure of the normal velocity $\tilde{w}(z)$ as $F \rightarrow 0$. In the appendix, we show how an approximation of the function $\Delta_{c}$ can be obtained using a WKBJ analysis. It is first shown that, when $F$ is small, $\Delta_{c}$ is in general exponentially small. Large values of $\Delta_{c}$ are only obtained when $\sin \alpha /(k F)$ is very close to $U_{0}$, as expected from figure 9 . This case corresponds to the limit (2). In this 


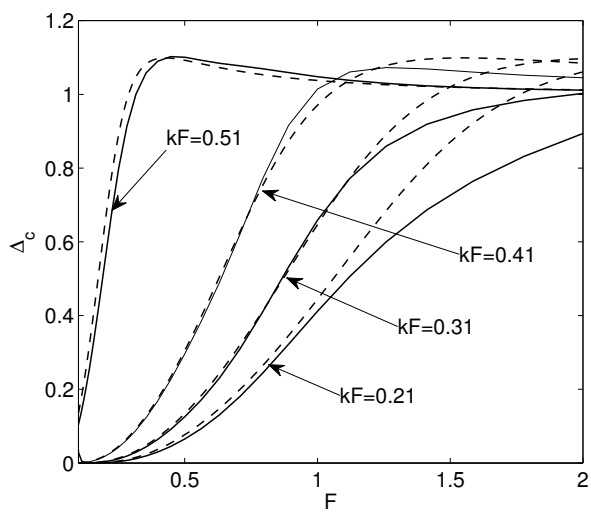

(a)

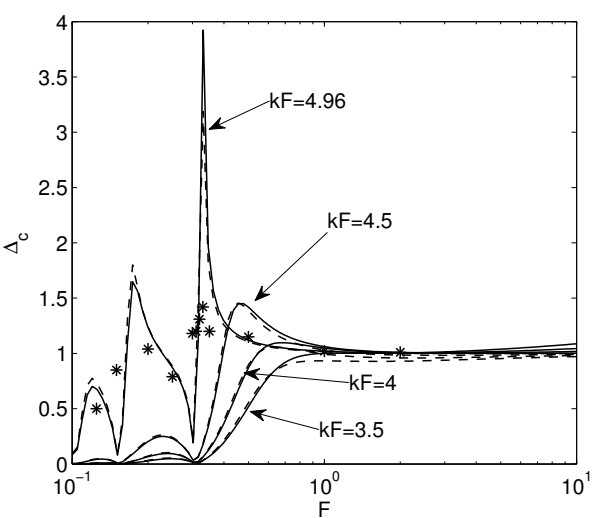

(b)

Figure 11. The function $\Delta_{c}$ versus $F$ for different values of $k F$ for (a) $\alpha=\pi / 60$ and (b) $\alpha=\pi / 6$. Solid lines: numerically computed values; Dashed lines: expression $(3.19 \mathrm{a}, \mathrm{b})$. The stars in (b) are estimates obtained from the direct numerical simulation for $k F=4.96, R e=593$ and $h=0.12$.

limit, a simple approximation is obtained for $\Delta_{c}$ as

$$
\begin{gathered}
\Delta_{c}=\frac{1}{X \sqrt{\left[K_{1}(X)\right]^{2}+\pi^{2}\left[I_{1}(X)\right]^{2}}} \text { when } k F<1 \\
\Delta_{c}=\frac{1}{X \sqrt{\left[K_{1}(X)+\frac{\pi}{2 \tan \Phi} I_{1}(X)\right]^{2}+\frac{\pi^{2}}{4}\left[I_{1}(X)\right]^{2}}} \text { when } k F>1,
\end{gathered}
$$

where $I_{1}$ and $K_{1}$ are modified Bessel functions and

$$
\begin{array}{r}
\Phi=k \int_{z_{c}}^{z_{\infty}} \sqrt{\frac{1-(k F)^{2} U^{2}(z)}{(k F)^{2} U^{2}(z)-\sin ^{2} \alpha}} d z, \\
X=\frac{\cos \alpha}{\left(1-U_{0}^{2}\right) F} \sqrt{2\left(1-\frac{k F U_{0}}{\sin \alpha}\right)} .
\end{array}
$$

The approximation depends on the value of $k F$ with respect to 1 because the structure of $\tilde{w}$ changes when $k F$ exceeds 1 . When $k F<1$, the solution is oscillating up to infinity, whereas, when $k F>1$ it becomes evanescent after the turning point $z_{\infty}$ where $k F U\left(z_{\infty}\right)=1$. The theoretical approximation is compared to the computed curve in figure 11. We can observe that it provides a good approximation even for $F$ as large as unity. In figure 11(b), we have also plotted the values provided by the direct numerical simulation for $k F=4.96, R e=596$ and $h=0.12$. Although the largest and smallest values of $\Delta_{c}$ are not recovered, we can observe that the oscillations of $\Delta_{c}$ as $F$ varies are qualitatively reproduced.

The behavior of $\Delta_{c}$ for $k F>1$ is particularly interesting as it exhibits distinct peaks and valleys. A simple estimate of $\Phi$ can be obtained in the limit of small $\alpha$ :

$$
\Phi \sim-\frac{\log (\alpha)}{F} .
$$

The valleys correspond to the configurations where $\tan \Phi=0$, that is $\Phi=n \pi, n=$ $1,2,3 \ldots$ For small $\alpha$, they are therefore obtained for fixed values of $F$ (whatever $k$ and 
$\left.U_{0}\right)$ given by

$$
F_{V}^{(n)}=-\frac{\log (\alpha)}{n \pi} .
$$

Present between each valley is a peak, which is reached (for small $\alpha$ ) for

$$
F_{P}^{(n)}=-\frac{\log (\alpha)}{n \pi-\arctan \left(\frac{\pi}{2} \frac{I_{1}(X)}{K_{1}(X)}\right)} .
$$

These peaks are the largest when $X$ is the smallest, that is when $k F$ is the closest to $\sin \alpha / U_{0}$. Their amplitude decreases as $X$ increases. This means that for a fixed value of $k F$, the amplitude of the peak decreases as $F$ decreases, as observed in figure 11.

In summary, when $F$ is small, we have shown that $\Delta_{c}$ (and thus also $v_{\max }$ ) reaches its largest values when $k F$ is slightly below $\sin \alpha / U_{0}$, that is when the critical point is close to the wall. When $k F>1$, a phenomenon of resonance and anti-resonance is observed generating large fluctuations of $\Delta_{c}$ as $F$ is varied. This phenomenon directly affects the maximum transverse velocity $v_{\max }$ which is proportional to $\Delta_{c}$ [see (3.14) and (3.15)]. For large $F$, we have seen that the largest values of $v_{\max }$ are obtained when $k F$ is slightly above $\sin \alpha$, that is when the critical point is far away from the wall.

Note however that when $F$ is of order $1, V$ remains $O(1)$. In that case, all the corrugation wavenumbers $k$ satisfying $(\sin \alpha) / F<k<(\sin \alpha) /\left(U_{0} F\right)$ are thus expected to give a large transverse flow response.

\section{Conclusion}

In this paper, we have presented some numerical and theoretical results on the internal waves generated by a sinusoidal topography on an inclined bottom. The velocity profile $U^{*}\left(z^{*}\right)$ has been chosen as an hyperbolic tangent profile, with a sliding velocity at the bottom. The inclination of the bottom has been found to create a large transverse velocity at the distance to the wall where the frequency of the topographic forcing $k^{*} U^{*}(z)$ is equal to the transverse buoyancy frequency $N^{*} \sin \alpha$ (in dimensional units). We have shown that this large amplitude is associated with a critical layer singularity of the inviscid solution. We have also shown that the solution can be regularized by performing a viscous critical layer analysis. An analytic solution has been obtained and found to be in good agreement with the numerical results. The maximum transverse velocity has been shown to be larger by a $R e^{1 / 3}$ factor - the normal velocity generated by the undulation at the wall. Interestingly, the transverse velocity has also been found to exhibit well-defined peaks associated with a quasi-resonance phenomenon for specific values of the Froude number when $k F$ is larger than 1 and close to $\sin \alpha / U_{0}$.

The analysis has been based on the hypothesis that the fluid slides on the boundary with a constant velocity. This sliding velocity guarantees that a non-negligible normal velocity is created by the undulations. This hypothesis implicitly assumes that the size of the undulations is larger than the viscous sublayer width. Note in particular that if the undulations were within the sublayer $\left(h \ll \boldsymbol{R} e^{-1 / 3}\right)$, the normal velocity would scale as $O\left(\boldsymbol{R} e^{-1 / 3}\right)$ and the maximum transverse velocity would then not be that large.

The present study demonstrates that the interplay between undulation and tilting could have a strong impact on the dynamics of stratified boundary layer flow. A small deformation on a weakly inclined bottom can generate a large transverse flow. This transverse flow is $O\left(\boldsymbol{R} \boldsymbol{e}^{1 / 3}\right)$ larger than the sliding velocity and localized in a $O\left(R e^{-1 / 3}\right)$ layer. For large Reynolds numbers, it therefore induces a strong transverse shear which 
may become unstable with respect to the Kelvin-Helmholtz instability. Such a mechanism has been observed in the dynamics of a tilted vortex (Boulanger et al. 2008). We suspect that it could be active in the boundary layer.

Note also that nonlinear effects are expected to arrive first in the critical layer. In particular, the critical layer is expected to become nonlinear as soon as the amplitude of the transverse velocity reaches $R e^{-1 / 3}$. It would therefore be interested to pursue the analysis in the nonlinear regime using the framework of the nonlinear critical layer theory (Benney \& Bergeron 1969; Haberman 1972).

The present mechanism of mixing is expected to be in competition with the mixing induced by the boundary layer instabilities on a flat wall. At least, two types of instability are expected to be present on a tilted wall for large Reynolds numbers: the viscous boundary layer instability associated with Tollmien-Schlichting modes (Wu \& Zhang $2008 a$ ), the radiative instability which is inviscid in nature and present only if the wall is inclined (Candelier et al. 2012). The conditions of appearance of these two instabilities in the parameter space $(\alpha, F, R e)$ are only partially known (Bai 2012) but both instabilities are expected to be present for the large Reynolds numbers of geophysical flows. It would thus be interesting to simulate a configuration where one of these instabilities is also present.

For very high Reynolds numbers, we expect the boundary layer to become turbulent. Yet, it is possible that the present mechanism could still be active in this regime. Indeed, a similar analysis can a priori be performed with a mean turbulent boundary layer profile and a turbulent eddy viscosity and it would lead to the same conclusion.

The phenomenon could then be present in real geophysical flows. For instance, taking values from the continental slope in Besio et al. (2004), the mean current flows velocity is $0.07 \mathrm{~cm} / \mathrm{s}$, the boundary layer thickness estimated at $10 \mathrm{~m}$, the mean Brunt-Väisälä at frequency measured at $N=0.024$ (van Haren \& Howarth 2004), we obtain $F=0.29$. For $U_{0}=0.1$ and a tilt angle close to tilted with an angle $\alpha=\pi / 90$ the present results tell us that all the corrugation wavelengths larger than $73 \mathrm{~m}$ will generate a response with a critical layer and therefore will be a source of transverse flow. Note that the wavelength of sand waves studied in Besio et al. (2004) appear to have a threshold close to a $100 \mathrm{~m}$ and height between 1 and $10 \mathrm{~m}$, which is consistent with the present study.

For a stably stratified atmospheric boundary layer flow, if we take the values reported by Frehlich et al. (2008), that is a nocturnal wind of velocity $10 \mathrm{~m} / \mathrm{s}$ with a boundary layer thickness $100 \mathrm{~m}$ in a stable stratification with a Brunt-Väisälä frequency equal to 0.03 $\mathrm{rad} / \mathrm{s}$, we get $F=3$. On a slope of 10 degrees (if $U_{0}$ is still equal to 0.1 ), we therefore expect all the corrugation wavelengths larger than $60 \mathrm{~m}$ to be active. For both cases, the Reynolds number is so large $\left(R e=10^{6}\right.$ and $R e=10^{8}$ for the current and the wind respectively) that even small corrugation amplitudes (of the order of $10 \%$ of the boundary layer thickness) generate transverse flows of the order of the mean flow (assuming $\Delta_{c}$ of order unity). Moreover, these flows are present in a very thin layer, which leads to strong transverse shears 2 orders of magnitude larger than the mean shear of the boundary layer. These shear layers are probably unstable by the Kelvin-Helmholtz instability and a non negligible source of mixing in the boundary layer. 


\section{Acknowledgements}

We would like to acknowledge Uwe Ehrenstein and Matthieu Marquillie for providing the unstratified code. This work has received a support from the French National

Research Agency under the A*MIDEX grant ANR-11-IDEX-0001-02, and is part of the LABEX MEC project ANR-11-LABX-0092.

\section{Appendix A. Small $F$ analysis of the equation for $\tilde{w}$}

In this section, we provide an asymptotic analysis in the limit of small $F$ of the solution to equation (3.8). Our objective is to obtain an estimate of the ratio $\Delta_{c}=\left|\tilde{w}_{c} / \tilde{w}_{0}\right|$ appearing in expression (3.15).

We assume that $\sin \alpha /(k F)=O(1)$ such that we can define the $O(1)$ parameter

$$
\alpha_{1}=\sin \alpha /(k F) .
$$

We also assume that there exists a critical point $z_{c}$ (different from the wall boundary) defined by

$$
U\left(z_{c}\right)=\alpha_{1}
$$

This implies that $\alpha_{1}$ satisfies $U_{\min }<\alpha_{1}<U_{\max }$, that is

$$
\sin \alpha<k F<\frac{\sin \alpha}{U_{0}} \text {. }
$$

\section{A.1. WKBJ analysis}

When $F \ll 1,(3.8)$ reduces to

$$
\tilde{w}^{\prime \prime}-\left(\frac{1-k^{2} F^{2} U^{2}}{F^{2}\left(\alpha_{1}^{2}-U^{2}\right)}+O(1)\right) \tilde{w}=0 .
$$

This equation is adequate for a resolution using WKBJ methods (see, for instance, Bender \& Orszag 1978). Far from the critical point, the solutions can be written at leading order as

$$
\tilde{w} \sim A \beta^{-1 / 4} \exp \left(\frac{1}{F} \int_{z_{c}}^{z} \sqrt{\beta}\right)+B \beta^{-1 / 4} \exp \left(-\frac{1}{F} \int_{z_{c}}^{z} \sqrt{\beta}\right),
$$

where

$$
\beta(z, k F)=\frac{1-(k F)^{2} U^{2}}{\alpha_{1}^{2}-U^{2}(z)} .
$$

Between 0 and $z_{c}, \beta$ is positive, so the solution is of exponential type. In this interval, the solution will then be dominated by one of the exponentials. It will be convenient to write it as

$$
\tilde{w} \sim \tilde{w}(0)\left(\frac{\beta(0)}{\beta}\right)^{1 / 4} \exp \left(-\frac{1}{F} \int_{0}^{z} \sqrt{\beta}\right) .
$$

By contrast, in the outer interval $\left(z_{c}, z_{\infty}\right)$, the solution is oscillatory and can be written as

$$
\tilde{w}^{+} \sim A(-\beta)^{-1 / 4} \exp \left(\frac{i}{F} \int_{z_{c}}^{z} \sqrt{-\beta}\right)+B(-\beta)^{-1 / 4} \exp \left(-\frac{i}{F} \int_{z_{c}}^{z} \sqrt{-\beta}\right) .
$$

If $k F<1$, the solution is oscillatory up to infinity $\left(z_{\infty}=\infty\right)$ : it must be an outgoing wave (as required by causality) which imposes $B=0$. If $k F>1, z_{\infty}$ is a finite location defined by $k F U\left(z_{\infty}\right)=1$. The solution becomes evanescent again after the turning point $z_{\infty}$. 
The condition of matching of an oscillating solution and an evanescent solution across a turning point is classical (see Bender \& Orszag 1978). It gives the condition:

$$
B=-i A \exp \left(\frac{2 i}{F} \int_{z_{c}}^{z_{\infty}} \sqrt{-\beta}\right) .
$$

In the following, we write $B=K_{ \pm} \mathrm{A}$ where $K_{-}$and $K_{+}$correspond to the constant when $k F<1$ and $k F>1$ respectively. We do not consider the special case $k F=1$ for which the turning point is at infinity.

The WKBJ approximations are not valid close to $z_{c}$. If $F^{2} R e^{1 / 3} \gg 1$, the region $\left|z-z_{c}\right|=O\left(F^{2}\right)$ is far away from the critical layer. In this region, $\tilde{w}$ is still given by (A 4) which now reduces at leading order to

$$
\tilde{w}^{\prime \prime}+\left(\frac{\cos ^{2} \alpha}{2 F^{2} \alpha_{1} U_{c}^{\prime}\left(z-z_{c}\right)}\right) \tilde{w}=0
$$

This equation admits the general solutions

$$
\begin{array}{r}
\tilde{w}=a^{+} \sqrt{\tilde{z}} J_{1}(\sqrt{\tilde{z}})+b^{+} \sqrt{\tilde{z}} Y_{1}(\sqrt{\tilde{z}}) \text { for } \quad \tilde{z}>0, \\
\tilde{w}=a^{-\sqrt{-\tilde{z}}} I_{1}(\sqrt{-\tilde{z}})+b^{-} \sqrt{-\tilde{z}} K_{1}(\sqrt{-\tilde{z}}) \text { for } \quad \tilde{z}<0,
\end{array}
$$

where

$$
\tilde{z}=\frac{2 \cos ^{2} \alpha}{F^{2} \alpha_{1} U_{c}^{\prime}}\left(z-z_{c}\right)
$$

The matching with the WKBJ approximations requires

$$
\begin{array}{r}
a^{-}=\frac{\sqrt{2 \pi \alpha_{1} U_{c}^{\prime} F}}{\cos \alpha\left(\alpha_{1}^{2}-U_{0}^{2}\right)^{1 / 4}} \tilde{w}(0) \exp \left(-\frac{1}{F} \int_{0}^{z_{c}} \sqrt{\beta}\right), \\
a^{+}=\frac{\sqrt{2 \pi \alpha_{1} U_{c}^{\prime} F}}{2 \cos \alpha} e^{3 i \pi / 4}\left(1+i K_{ \pm}\right) A, \\
b^{+}=\frac{\sqrt{2 \pi \alpha_{1} U_{c}^{\prime} F}}{2 \cos \alpha} e^{-3 i \pi / 4}\left(1-i K_{ \pm}\right) A .
\end{array}
$$

The solutions (A 11a,b) are not regular at $z_{c}$. Close to $z_{c}$, (A 11a,b) expand as

$$
\begin{gathered}
\tilde{w} \sim a^{+} \frac{\tilde{z}}{2}-\frac{2 b^{+}}{\pi}\left(1-\frac{\tilde{z}(\ln (\tilde{z})-2 \ln (2)-1+2 \gamma)}{4}\right), \text { for } \tilde{z}>0, \\
\tilde{w} \sim-a^{-} \frac{\tilde{z}}{2}+b^{-}\left(1-\frac{\tilde{z}(\ln (-\tilde{z})-2 \ln (2)-1+2 \gamma)}{4}\right), \text { for } \tilde{z}<0 .
\end{gathered}
$$

This critical layer singularity can only be smoothed by introducing viscous effects. However, this is not necessary for obtaining the relations between $a^{-}, b^{-}$and $a^{+}, b^{+}$. Indeed, when $F^{2} R e^{1 / 3} \gg 1$, the solution is expected to remain regular on a contour that avoids the singularity in the lower complex $\tilde{z}$-plane. This condition means that (A 14b) should correspond to (A 14a) where $-\tilde{z}$ has been changed into $\tilde{z} e^{-i \pi}$ in the logarithm. This yields the following conditions:

$$
\begin{aligned}
b^{-} & =-\frac{2}{\pi} b^{+}, \\
a^{-}+a^{+} & =-\frac{i \pi}{2} b^{-} .
\end{aligned}
$$

Note that these conditions could have been directly obtained by requiring (A 11a) to be valid for $-\pi \leqslant \arg (\tilde{z}) \leqslant 0$. This would have implied that (A 11a) is valid for negative $\tilde{z}$ 
with $\sqrt{\tilde{z}}$ defined as $-i \sqrt{-\tilde{z}}$ for negative $\tilde{z}$. The equations (A 13a-c) and (A 15a,b) give a relation between $\tilde{w}(0)$ and $\tilde{w}\left(z_{c}\right)=b^{-}$which can be written as

$$
\tilde{w}\left(z_{c}\right)=\tilde{w}(0) K_{o \pm} \sqrt{F} \exp \left(-\frac{1}{F} \int_{0}^{z_{c}} \sqrt{\beta}\right),
$$

where $K_{o \pm}$ is a constant independent of $F$ :

$$
K_{o \pm}=\frac{\left(1-i K_{ \pm}\right) \sqrt{2 \pi \alpha_{1} U_{c}^{\prime}}}{\pi\left(\alpha_{1}^{2}-U_{0}^{2}\right)^{1 / 4} \cos \alpha} e^{i \pi / 4}
$$

Expression (A 16) demonstrates that $\left|\tilde{w}_{c} / \tilde{w}_{0}\right|$ is in general exponentially small. There is an important exception which occurs when $z_{c}$ is close to the wall.

\section{A.2. Special case of the critical point close to the wall}

We are going to see that large values of $\left|\tilde{w}_{c} / \tilde{w}_{0}\right|$ can be obtained when $z_{c}=O\left(F^{2}\right)$. This condition requires that $\alpha_{1}-U_{0}=O\left(F^{2}\right)$, such that

$$
z_{c} \approx\left(\alpha_{1}-U_{0}\right) / U_{0}^{\prime}=\left(\alpha_{1}-U_{0}\right) /\left(1-U_{0}^{2}\right) .
$$

The local solution is then valid between 0 and $z_{c}$ and can be written for $\tilde{z}<0$ as

$$
\tilde{w}=\left(1+i K_{ \pm}\right) \sqrt{-\tilde{z}} J_{1}(-i \sqrt{-\tilde{z}})+i\left(1-i K_{ \pm}\right) \sqrt{-\tilde{z}} Y_{1}(-i \sqrt{-\tilde{z}}) .
$$

Therefore the ratio $\Delta_{c}=\left|\tilde{w}\left(\tilde{z}_{c}\right) / \tilde{w}(0)\right|$ satisfies

$$
\begin{gathered}
\Delta_{c}^{-}=\frac{2}{\pi X\left|J_{1}(-i X)+i Y_{1}(-i X)\right|} \text { when } k F<1, \\
\Delta_{c}^{+}=\frac{2}{\pi X\left|J_{1}(-i X) / \tan \Phi+Y_{1}(-i X)\right|} \text { when } k F>1,
\end{gathered}
$$

where

$$
\begin{array}{r}
\Phi=\frac{1}{F} \int_{z_{c}}^{z_{\infty}} \sqrt{-\beta}, \\
X=\sqrt{-\tilde{z}_{c}}=\sqrt{\frac{2\left(\alpha_{1}-U_{0}\right) \cos ^{2} \alpha}{\left(1-U_{0}^{2}\right)^{2} F^{2} \alpha_{1}}} .
\end{array}
$$

The functions $\Delta_{c}^{ \pm}$can also be written as

$$
\begin{gathered}
\Delta_{c}^{-}=\frac{1}{X \sqrt{\left[K_{1}(X)\right]^{2}+\pi^{2}\left[I_{1}(X)\right]^{2}}}, \\
\Delta_{c}^{+}=\frac{1}{X \sqrt{\left[K_{1}(X)+\frac{\pi}{2 \tan \Phi} I_{1}(X)\right]^{2}+\frac{\pi^{2}}{4}\left[I_{1}(X)\right]^{2}}} .
\end{gathered}
$$

When $k F<1$, the maximum of $\Delta_{c}^{-}$is $\Delta_{c \max }^{-} \approx 1.1$, reached for $X \approx 0.42$; when $k F>1$, the maximum of $\Delta_{c}$ is not bounded. For a fixed $X$, its maximum value, reached when

$$
\tan \Phi_{\max }=-\frac{\pi}{2} \frac{I_{1}(X)}{K_{1}(X)}
$$

is

$$
\Delta_{c \max }^{+}(X)=\frac{2}{\pi X I_{1}(X)} .
$$

This maximum diverges as $4 /\left(\pi X^{2}\right)$ for small $X$. For a fixed $\Phi, \Delta_{c}^{+}$exhibits a maximum 


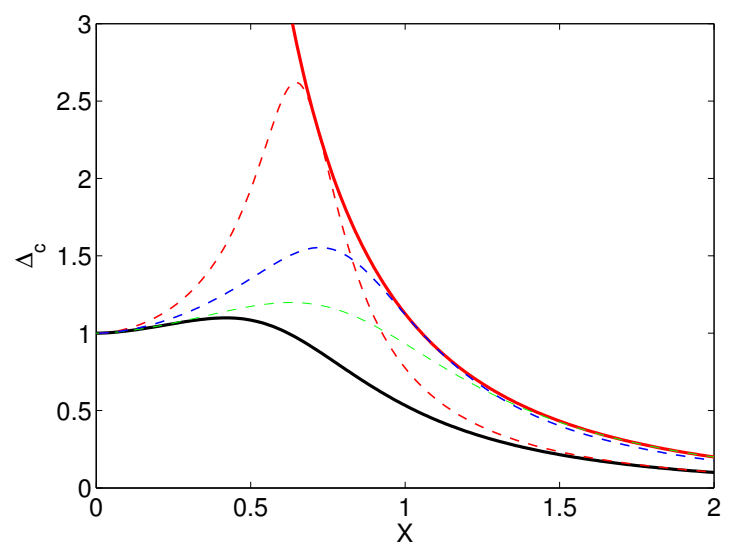

FiguRE 12. Function $\Delta_{c}=\left|\tilde{w}\left(\tilde{z}_{c}\right) / \tilde{w}(0)\right|$ versus $X$. The solid black line is $\Delta_{c}^{-}(X)$, that is for $k F<1$. The solid red line is $\Delta_{c \max }^{+}(X)$ (maximum value of $\Delta_{c}^{+}$over all $\Phi$ for $k F>1$ ). The dotted lines are $\Delta_{c}^{+}$for $\Phi=\pi / 2$ (green), $\Phi=2 \pi / 3$ (blue) and $\Phi=5 \pi / 6$ (red).

for a value $X^{\max }$ smaller than 0.73 . This maximum is the largest when $\Phi$ is slightly below a multiple of $\pi$. In that case, $X^{\max }$ is very close to 0 . On the opposite, when $\Phi$ is exactly equal to a multiple of $\pi$, the function $\Delta_{c}^{+}$vanishes for all $X>0$.

The function $\Delta_{c}$ is plotted for both cases $k F<1$ and $k F>1$ in figure 12 .

\section{REFERENCES}

Abramowitz, M. \& Stegun, I. A. 1965 Handbook of Mathematical Functions. New York: Dover.

ACheson, D. J. 1976 On over-reflection. J. Fluid Mech. 77, 433-472.

Athanassiadou, M. \& Castro, I. P. 2001 Neutral flow over a series of rough hills: a laboratory experiment. Boundary-Layer Met. 101, 1-30.

BAI, Y. 2012 Rayonnement d'une couche limite dans un milieu stratifié. Master's thesis, University Paris XI.

Bender, C. M. \& Orszag, S. A. 1978 Advanced mathematical methods for scientists and engineers. New York: McGraw-Hill.

Benney, D. J. \& Bergeron, R. F. 1969 A new class of nonlinear waves in parallel flows. Stud. Appl. Math. 48, 181-204.

Besio, G., Blondeaux, P., M., Brocchini \& Vittori, G. 2004 On the modeling of sand wave migration. J. Geophys. Res. 109, 1-13.

Boulanger, N., Meunier, P. \& Le Dizès, S. 2007 Structure of a tilted stratified vortex. $J$. Fluid Mech. 583, 443-458.

Boulanger, N., Meunier, P. \& Le Dizès, S. 2008 Instability of a tilted vortex in stratified fluid. J. Fluid Mech. 596, 1-20.

Candelier, J., Le Dizès, S. \& Millet, C. 2012 Inviscid instability of a stably stratified compressible boundary layer on an inclined surface. J. Fluid Mech. 694, 524-539.

Drazin, P. G. \& Reid, W. H. 1981 Hydrodynamic stability. Cambridge University Press.

Finnigan, J. J., Shaw, R. H. \& Patton, E. G. 2009 Turbulence structure above a vegetation canopy. J. Fluid Mech. 637, 387-424.

Frehlich, R., Meillier, Y. \& Jensen, M. L. 2008 Measurements of boundary layer profiles with in situ sensors and doppler lidar. J. Atmos. Ocean. Tech. 25, 1328-40.

Garratt, J. 1992 The Atmospheric Boundary Layer. Cambridge Univ. Press.

Garrett, C., MacCready, P. \& Rhines, P. 1993 Boundary mixing and arrested Ekman layers: rotating stratified flow near a sloping boundary. Annu. Rev. Fluid Mech. 25, 291323. 
Genin, A., Dayton, P. K., Lonsdale, P. F. \& Spiess, F. N. 1986 Corals on seamount peaks provide evidence of current acceleration over deep sea topography. Nature 322, 59-61.

Gong, W., Taylor, P. A. \& Dornbrack, A. 1996 Turbulent boundary-layer flow over fixed aerodynamically rough two-dimensional sinusoidal waves. J. Fluid Mech. 312, 1-31.

Haberman, R. 1972 Critical layers in parallel flows. Stud. Appl. Math. 51, 139-161.

VAN HAREN, H. \& Howarth, M. J. 2004 Enhanced stability during reduction of stratification in the north sea. Cont. Shelf Res. 24, 805-819.

Jackson, S. \& Hunt, J. C. R. 1975 Turbulent wind flow over a low hill. Q. J . R. Met. Soc. 101, 929-955.

Lin, C. C. 1955 The theory of hydrodynamics stability. Cambridge University Press.

LindzEn, R. S. \& BARKER, J. W. 1985 Instability and wave over-reflection in stably stratified shear flow. J. Fluid Mech. 151, 189-217.

MacCready, P. \& Pawlak, G. 2001 Stratified flow along a rough slope: Separation drag and wave drag. J. Phys. Oceanogr. 31, 2824-2839.

Mahrt, L. 2014 Stably stratified atmospheric boundary layers. Annu. Rev. Fluid Mech. 46, $23-45$.

Marquillie, M. \& Ehrenstein, U. 2002 Numerical simulation of a separating boundary-layer flow. Compt. and Fluids 31, 683-693.

Marquillie, M. \& Ehrenstein, U. 2003 On the onset of nonlinear oscillations in a separating boundary-layer flow. J. Fluid Mech. 490, 169-188.

Nadeau, D. F., Pardyjak, E. R., Higgins, C. W. \& Parlange, M. B. 2013 Similarity scaling over a steep alpine slope. Boundary-Layer Met. 147, 401-419.

OнуA, Y. 2001 Wind-tunnel study of atmospheric stable boundary layers over a rough surface. Boundary-Layer Met. 98, 57-82.

OhyA, Y. \& UChidA, T. 2008 Laboratory and numerical studies of the atmospheric stable boundary layers. J. Wind Eng. Ind. Aerodyn. 96, 2150-2160.

PARK, M. S. \& PARK, S. U. 2006 Effects of topographical slope angle and atmospheric stratification on surface-layer turbulence. Boundary-Layer Met. 147, 613-633.

Passaggia, P.-Y., Leweke, T. \& Ehrenstein, U. 2012 Transverse instability and lowfrequency flapping in separated boundary-layer flows: An experimental study. J. Fluid Mech. 703, 363-373.

Riedinger, X., Le Dizès, S. \& Meunier, P. 2011 Radiative instability of the flow around a rotating cylinder in a stratified fluid. J. Fluid Mech. 672, 130-146.

Saric, W. S., Reed, H. L. \& Kerschen, E. J. 2002 Boundary-layer receptivity to freestream disturbances. Annu. Rev. Fluid Mech. 34, 291-319.

Sykes, R. I. 1978 Stratification effects in boundary layer flow over hills. Proc. Roy. Soc. Lond. A 361, 225-243.

Taylor, P. A., Mason, P. J. \& Bradley, E. F. 1987 Boundary-layer flow over low hills. Boundary-Layer Met. 39, 107-132.

Thorpe, S. A. 1992 The generation of internal waves by flow over the rough topography of a continental slope. Proc. Roy. Soc. Lond. A 439, 115-130.

Wu, X. \& ZhANG, J. 2008 a Instability of a stratified boundary layer and its coupling with internal gravity waves. Part 1. Linear and nonlinear instabilities. J. Fluid Mech. 595, $379-408$.

Wu, X. \& Zhang, J. $2008 b$ Instability of a stratified boundary layer and its coupling with internal gravity waves. Part 2. Coupling with internal gravity waves via topography. $J$. Fluid Mech. 595, 409-433. 